\section{Reply to H.J.A. Adams et al and C. Kobe et al}

We thank Adams and Kwee ${ }^{1}$ and Kobe et $\mathrm{al}^{2}$ for their interest in our subsidiary analysis from the RAPID (Randomized Phase III Trial to Determine the Role of Fluorodeoxyglucose-PET Imaging in Clinical Stages IA/IIA Hodgkin Disease) trial. ${ }^{3}$ In this study, we demonstrate that individual positron emission tomography (PET) score is strongly associated with outcome. Patients with stage IA to IIA early-stage Hodgkin lymphoma $(\mathrm{HL})$ with a PET score of 5 after three cycles of doxorubicin, bleomycin, vinblastine, and dacarbazine (ABVD) had significantly worse outcomes, with 5-year event-free survival (EFS) and overall survival rates of $61.9 \%$ (95\% $\mathrm{Cl}, 41.1 \%$ to $82.7 \%)$ and $85.2 \%(95 \% \mathrm{Cl}, 69.7 \%$ to $100 \%)$, respectively, compared with $93.4 \%(95 \% \mathrm{Cl}$, $91.0 \%$ to $95.8 \%)$ and $97.8 \%(95 \% \mathrm{Cl}, 96.4 \%$ to 99.2\%) in patients with PET scores of 1 to 4.

Adams and $\mathrm{Kwee}^{1}$ conclude rightly that a majority of patients with early-stage HL have reasonable outcomes without treatment escalation and highlight that the proportion of patients in our study with a PET score of 5 was low. However, we strongly disagree that these markedly inferior EFS and overall survival rates constitute reasonable outcomes for patients with a PET score of 5 treated with four cycles of ABVD and involved-field radiotherapy, particularly when the potential benefits of early treatment intensification have already been demonstrated by the $\mathrm{H} 10$ trial. ${ }^{4}$

Kobe et $\mathrm{al}^{2}$ raise concerns that our results were biased by treatment differences. However, patients with a score of 5 had significantly higher risk than those with all other scores, including patients with a score of 3 or 4 who received identical treatment. The strong association between a score of 5 and inferior outcome is a robust finding, which remained after adjusting for treatment in patients with a score of 1 or 2 and excluding patients who did not receive radiotherapy. ${ }^{3}$

We thank Kobe et $\mathrm{al}^{2}$ for highlighting inconsistencies in the definition of Deauville score 5 (DS5) and providing the opportunity to clarify the current internationally agreed recommendations. ${ }^{5,6}$ The 5-point scale was developed by our group ${ }^{7}$ and adopted as the preferred reporting method at the first international workshop in Deauville. ${ }^{8}$ A score of 5 was originally defined by us as "uptake markedly higher than the liver and/or new lesions." "7(p1826;3050-3051) We chose to use three times the maximum liver uptake in RAPID, ${ }^{3,9}$ with appropriately rigorous quality control to ensure quantitative accuracy. The Deauville publication defined DS5 as "markedly increased uptake at any site and new sites of disease," ${ }^{(p 1259)}$ but this was subsequently revised in line with our original definition, and the original definition was used in validation studies ${ }^{10,11}$ and 2014 international guidance. ${ }^{5,6}$

The decision-making scan in RAPID was not an endof-treatment assessment for most patients., 3,9 The 2014 guidance stated an interim score of 4 or 5 may represent chemotherapy-sensitive disease if "uptake has reduced from baseline" ${ }^{\text {(p3062) }}$ but that the degree of reduction that predicted adequate response was "dependent on disease type, timing, and treatment given." ${ }^{(\text {(р3063) }}$ We do not seek to redefine existing definitions of PET positivity, which apply primarily to end of treatment, but rather to provide important data where evidence is lacking regarding use of interim PET.

We appreciate concerns raised by Kobe et al ${ }^{2}$ that our results should not be overinterpreted. We are conscious of the limitations of this exploratory analysis, clearly acknowledging that "relatively few patients had a PET score 4 with a small number of events." ${ }^{3}$ We have not claimed outcomes to be equivalent for patients with scores of 1 to 3 and a score of 4; however, we did not observe a difference between these groups. Although EFS was inferior for patients with a score of 4 or 5 combined, this was driven almost entirely by events in patients with a score of 5. Patients with a score of 4 had good outcomes with ABVD and involved-field radiotherapy, with a 5-year EFS of $93.5 \%(95 \% \mathrm{Cl}, 84.9 \%$ to $100 \%)$. Treatment of patients with DS4 and nonbulky early-stage $\mathrm{HL}$ requires careful consideration in light of the $\mathrm{H} 10$ study, ${ }^{4}$ but our results highlight uncertainty regarding the need for treatment escalation in this group. ${ }^{3}$

Adams and Kwee ${ }^{1}$ point out that most relapses occurred in patients with scores of 1 to 4 and conclude that these patients cannot benefit from PET-guided approaches. However, in the $93.4 \%(95 \% \mathrm{Cl}, 91.0 \%$ to $95.8 \%)$ of patients with PET scores of 1 to 4 who remained event free at 5 years, excellent outcomes were achieved with less-intensive treatment than with the prior standard of care and the $\mathrm{H} 10$ approach ${ }^{4}$ in patients with nonbulky stage I to IIA HL. We therefore believe that there are clear benefits with PET-guided treatment.

We thank Kobe et $\mathrm{al}^{2}$ for acknowledging the importance of early PET response in risk-adapted treatment strategies and agreeing that our results are hypothesis generating. We also believe that our findings emphasize the need to re-evaluate clinical prognostic groupings. We hope this subsidiary analysis ${ }^{3}$ will assist in future trial designs and promote reporting of individualized PET scores using clear methodology to enable external validation, while supporting a need for treatment intensification in patients with DS5.

\section{Sally F. Barrington, MBBS, MSc}

Kings College London, London, United Kingdom 
Elizabeth H. Phillips, MB and Nicholas Counsell, MSc University College London, London, United Kingdom

John Radford, MD

University of Manchester, Manchester, United Kingdom

AUTHORS' DISCLOSURES OF POTENTIAL CONFLICTS OF INTEREST AND DATA AVAILABILITY STATEMENT

Disclosures provided by the authors and data availability statement (if applicable) are available with this article at DOI https://doi.org/10.1200/ JC0.19.02056

\section{ACKNOWLEDGMENT}

The RAPID trial was supported by the Leukaemia and Lymphoma Research Fund (now Bloodwise), the Lymphoma Research Trust, Teenage Cancer Trust, and the UK Department of Health and run by the Cancer Research UK (CRUK) and University College London Cancer Trials Centre. S.F.B. is supported by National Institute for Health Research and Social Care (NIHR) Grant No. RP-2-16-07-001. The King's College London and University College London Comprehensive Cancer Imaging Centre is funded by the CRUK and Engineering and Physical Sciences Research Council in association with the Medical Research Council and Department of Health (England). The views expressed are those of the authors and not necessarily those of the National Health Service, NIHR, or Department of Health. We thank the investigators, positron emission tomography centers, and patients from all parts of the United Kingdom for their support.

\section{REFERENCES}

1. Adams HJA, Kwee TC: Only a small proportion of early-stage Hodgkin lymphoma patients may potentially benefit from fluorodeoxyglucosepositron emission tomography-adapted treatment escalation. J Clin Oncol 37:3323, 2019

2. Kobe $\mathrm{C}$, Goergen $\mathrm{H}$, Fuchs $\mathrm{M}$, et al: Predictive value of positron emission tomography/computed tomography after ABVD-based chemotherapy in early-stage Hodgkin Iymphoma. J Clin Oncol 37: 3324-3325, 2019

3. Barrington SF, Phillips EH, Counsell N, et al: Positron emission tomography score has greater prognostic significance than pretreatment risk stratification in early-stage Hodgkin lymphoma in the UK RAPID study. J Clin Oncol 37:1732-1741, 2019

4. André MPE, Girinsky T, Federico M, et al: Early positron emission tomography response-adapted treatment in stage I and II Hodgkin lymphoma: Final results of the randomized EORTC/LYSA/FIL H1O trial. J Clin Oncol 35:1786-1794, 2017

5. Barrington SF, Mikhaeel NG, Kostakoglu L, et al: Role of imaging in the staging and response assessment of lymphoma: Consensus of the International Conference on Malignant Lymphomas Imaging Working Group. J Clin Oncol 32:3048-3058, 2014

6. Cheson BD, Fisher RI, Barrington SF, et al: Recommendations for initial evaluation, staging, and response assessment of Hodgkin and nonHodgkin lymphoma: The Lugano classification. J Clin Oncol 32:3059-3068, 2014

7. Barrington SF, Qian W, Somer EJ, et al: Concordance between four European centres of PET reporting criteria designed for use in multicentre trials in Hodgkin lymphoma. Eur J Nucl Med Mol Imaging 37:1824-1833, 2010

8. Meignan M, Gallamini A, Meignan M, et al: Report on the first international workshop on interim-PET-scan in Iymphoma. Leuk Lymphoma 50: 1257-1260, 2009

9. Radford J, Illidge T, Counsell N, et al: Results of a trial of PET-directed therapy for early-stage Hodgkin's lymphoma. N Engl J Med 372: 1598-1607, 2015

10. Biggi A, Gallamini A, Chauvie $S$, et al: International validation study for interim PET in ABVD-treated, advanced-stage Hodgkin lymphoma: Interpretation criteria and concordance rate among reviewers. J Nucl Med 54:683-690, 2013

11. Itti E, Meignan M, Berriolo-Riedinger A, et al: An international confirmatory study of the prognostic value of early PET/CT in diffuse large B-cell lymphoma: Comparison between Deauville criteria and $\Delta$ SUVmax. Eur J Nucl Med Mol Imaging 40:1312-1320, 2013

DOI: https://doi.org/10.1200/JC0.19.02056; Published at jco.org on September 30, 2019. 
AUTHORS' DISCLOSURES OF POTENTIAL CONFLICTS OF INTEREST

Reply to H.J.A. Adams et al and C. Kobe et al

The following represents disclosure information provided by authors of this manuscript. All relationships are considered compensated. Relationships are self-held unless noted. I = Immediate Family Member, Inst = My Institution. Relationships may not relate to the subject matter of this manuscript. For more information about ASCO's conflict of interest policy, please refer to www.asco.org/rwc or ascopubs.org/jco/site/ifc.

Sally F. Barrington

Speakers' Bureau: F. Hoffmann-La Roche

Research Funding: Bristol-Myers Squibb (Inst), Celgene (Inst), F. Hoffmann-La Roche (Inst), Amgen (Inst)

Elizabeth H. Phillips

Research Funding: F. Hoffmann-La Roche (Inst)
John Radford

Stock and Other Ownership Interests: GlaxoSmithKline (I), AstraZeneca (I)

Honoraria: Takeda Pharmaceuticals

Consulting or Advisory Role: Takeda Pharmaceuticals, Seattle Genetics,

Novartis

Speakers' Bureau: Takeda Pharmaceuticals, Seattle Genetics, Novartis

Research Funding: Takeda Pharmaceuticals

Travel, Accommodations, Expenses: Takeda Pharmaceuticals, ADC

Therapeutics

No other potential conflicts of interest were reported. 